\title{
HYBRID SCHEDULING FRAMEWORK FOR PARALLEL VISUALIZATION OF LARGE-SCALE MULTIPHYSICS SIMULATION DATA
}

\author{
Yi Cao, Zeyao Mo, Zhiwei Ai, Huawei Wang and Li Xiao \\ High Performance Computing Center \\ Institute of Applied Physics and Computational Mathematics \\ Beijing, China
}

\begin{abstract}
Following the recent rapid growth in supercomputer performance, many real-world problems in fields such as climate change and weather forecasting, nuclear energy, and electromagnetic environments can be solved via multiphysics simulation. However, current multifield visualization has difficulty handling multiphysics parallel simulation data. First, it is difficult to correctly visualize overlapping multifield data with semitransparent properties because of the complex distribution of partitioned data domains across multicore processors. Second, the interactive visualization performance of large-scale multifield data in serial processing mode on a personal computer is often slow because multiphysics simulations can produce large-scale data sets, i.e., of the order of gigabytes to terabytes. This paper introduces a hybrid scheduling framework for parallel visualization of computational multifield data, which is used to overcome problems both in correct visual representation and in efficient visualization of large-scale computational multifield data. The proposed framework supports scalable in situ visualization of 8.5 billion mesh cells on the $10 \mathrm{k}$ cores of China's TianHe-2 supercomputer, which could help domain scientists understand multiphysics phenomena more clearly and with greater accuracy.
\end{abstract}

\section{KEYWORDS}

Multiphysics, Multifield Visualization, Parallel Visualization, In Situ Visualization

\section{INTRODUCTION}

In real-world applications, simulation of large numbers of complex physical phenomena that are usually composed of various tightly coupled physical processes is known as multiphysics simulation. Multiphysics simulation can reveal new physical phenomena that cannot be elucidated via single physics simulation in the fields of science and engineering. Following the recent rapid growth of supercomputer performance, many multimaterial, multiscale, and multidiscipline real-world problems can now be overcome using multiphysics simulation. The demand for visualization of coupled multiphysics fields has emerged in several important application domains, e.g., climate change, weather forecasting, electromagnetic (EM) environments, and nuclear energy. The data sets output by such applications usually contain physical multifield data such as scalar fields, vector fields, or tensor fields that describe multiphysics processes. However, most existing research in the visualization community is limited to single-field visualization problems. Multifield visualization currently faces two considerable challenges. First, it is difficult to correctly visualize overlapping multifield data with semitransparent properties because of the complex distribution of partitioned data domains across multicore processors. Second, the interactive visualization performance of large-scale multifield data in serial processing mode on a personal computer is slow.

To help domain scientists understand accurately and efficiently the complex phenomena behind multifield data, we present a scalable parallel visualization framework for large-scale computational multifield data. The main contributions of this paper comprise the following:

- A multiplex visual-channels-based visualization strategy to encode multifield data based on a proper combination of visual channels that could enable scientists to correctly decode phenomena from the resultant images. 
- A hybrid scheduling framework for parallel visualization that improves the efficiency of large-scale multifield visualization, by taking advantage of data locality within multiple visual channels.

This parallel visualization framework improves the interactive visual analysis of computational multifield data in fields such as fusion energy and EM environments, supporting scalable in situ visualization of 8.5 billion mesh cells under the $10 \mathrm{k}$ cores of China's TianHe-2 supercomputer. This framework can satisfy the larges-scale visualization needs of typical multiphysics simulation applications.

\section{RELATED WORK}

In the field of science and engineering computing, multiphysical simulation is involved in many complex applications (Lethbridge, 2015), e.g., fusion and fission energy, EM environments, global climate change, and weather forecasting. Most of these typical applications still use traditional single-field visualization methods to support multifield data analysis because of the limitations of multifield visualization. Ensemble visualization (Phadke et al., 2012) supports exploration and comparison of a collection of single-field data produced by simulations for the same physical phenomenon with different initial conditions or parameters. Furthermore, constrained by the interactive performance of large-scale data analysis, domain-driven applications rely primarily on 2D cross-section based visualization to reveal spatial trends in 3D space.

Volume rendering is one of the most difficult methods to implement in multifield visualization. Multifield data require a stage of visual fusion within the visualization pipeline. Traditional rendering methods only support visual fusion between volume data and opaque geometric data (Kreeger et al., 1999; Insley et al., 2011). Jacq et al. (1997) mapped the attributes of multifield data into color space and implemented fusion at the color level. However, the new color generated by color fusion tends to confuse the visualized results. Translucent fusion based on the ray-accumulation-level method can represent more accurately the depth relationship (Cai et al., 1999), but it relies on visually accurate sorting of all the objects within the scene.

Existing sorting methods have certain limitations in terms of both fidelity and efficiency. (1) Z-buffering (Eberly, 2006) does not support sorting between translucent objects. (2) The painter algorithm (Porter et al., 1984) cannot handle the sort of intersecting objects with ambiguity. (3) Backface culling (Eberly, 2006) cannot be used to sort concave objects. (4) Depth peeling involves high computational complexity of the algorithm and relies on hardware acceleration. 5) Mesh subdivision relies on a large number of newly generated convex mesh cells for overlapping mesh regions, which makes it unsuitable for large-scale multifield data visualization. Considering the fidelity and efficiency of visualization, a new parallel visualization framework is required for multiphysics simulation data that can maintain the original mesh data generated by the multiphysics simulation.

\section{COMPLEX MESH SYSTEMS IN MULTIPHYSICS SIMULATION}

Popular parallel visualization system for scientific data considers only simple types of mesh systems, e.g., convex meshes or domains, in which each domain consists of one or more mesh cells. However, mesh data of multiphysics simulations are more complicated. Some typical mesh data characteristics of multiphysics simulations are listed below.

\subsection{Irregular and Adaptive Mesh System}

Computational problem solving for real-world applications often involves complex boundary conditions of physical models. Therefore, the numerical solver of an application often uses a complex mesh system with specific characteristics (e.g., irregular and adaptive meshes) for improved adaptation to complex boundary problems. Often, these types of mesh system present complex domain features such as concavities and holes. 


\subsection{Domain Decomposition}

Domain decomposition is also often used to solve boundary value problems in numerical partial differential equations. This method can decompose a complex boundary value problem into a boundary value problem of a smaller subdomain, which is solvable using adjacent subregions. As problem solving for such subdomains is reasonably independent, it is highly suitable for parallel computing. However, the region shapes of the decomposed domain might become more complex. For example, an original convex domain could be decomposed to several concave subdomains.

\subsection{Load Balancing}

Applications in the field of parallel computing often use static or dynamic load balancing to achieve efficient large-scale numerical simulation. However, load balancing can change the connectivity of the original problem domain. For example, if a parallel process is newly allocated mesh data from another process during the load-balancing strategy, both the newly allocated and the original mesh data on this parallel process might constitute a new complex domain with concave, disconnected, or non-manifold features. Figure 1 shows the load distribution of a multiphysics simulation in the field of laser plasma interaction (Ren et al., 2012). Mesh cells with the same color are assigned to the same processor core. It can be seen that many domains distributed on same processor core have concave or even disconnected features.

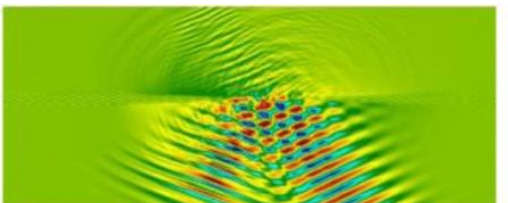

(a) Distribution of electric field intensity

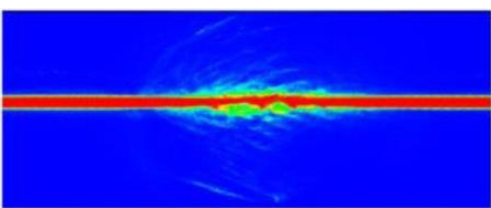

(c) Distribution of particle density

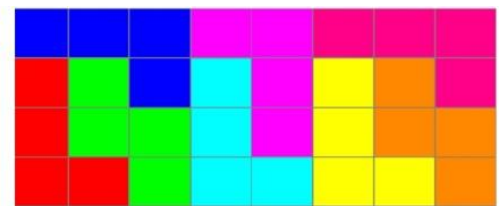

(b) Distribution of load balancing

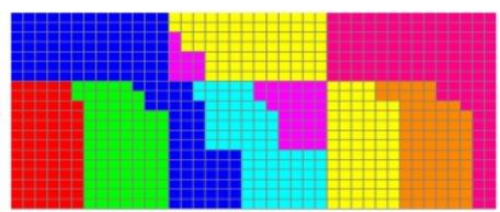

(d) Distribution of load balancing

Figure 1. Complex distribution of Mesh Data in Laser Plasma Interaction Simulation

\section{HYBRID SCHEDULING FRAMEWORK FOR PARALLEL VISUALIZATION}

\subsection{Multiplex Visual Channels Based Multifield Visualization}

For visual representation of computational multifield data, we introduced multiplex visual channels (Bertin, 1983). Taking advantage of the multichannel characteristics of human vision system (HVS, 2019), we consider multifield visualization as a coding process. By properly encoding multifield data through a combination of multiple visual channels, domain experts could correctly decode phenomena from resultant images. The visual channels defined in the proposed framework are divided into two types: geometric and optical. Figure 2 represents multifield data containing four different physics fields and the operations that act between them. The geometric channels, which provide a means for distinguishing visual objects based on geometric properties, are designed for dealing only with opaque geometry. The optical channels provide a means for distinguishing visual objects based on optical effects. Different operation of visual fusion is defined in different channels, which can be used to separate opaque and transparent objects during 
visualization. For example, the rendering of opaque objects in geometric channels only needs a simple depth comparison test, whereas the rendering of translucent objects in the optical channels uses the over operator introduced in the painter's algorithm.

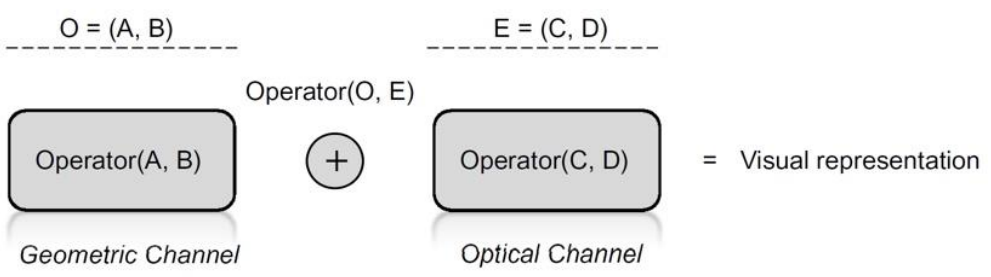

Figure 2. Multiplex Visual Channels for Multifield Visualization

\subsection{General Parallel Rendering}

A parallel visualization pipeline usually employs a five-phase process: data partitioning, data distribution, rendering, image composition, and display. The domain decomposition strategy of the numerical simulation determines how the global data are divided and distributed. Although visualization can regenerate the partitioned data that are more suitable for visualization, it is not feasible for large-scale simulation applications. Therefore, the parallel visualization pipeline discussed in this paper does not consider changing the original data partitioning.

For general parallel rendering (Molnar et al., 1994), the rendering is considered a sort problem based on screen space. Mainstream visualization systems often use sort-last rendering to achieve efficient visualization. Because the sort object of sort-last rendering is pixel-level data, it can only provide correct rendering when each domain distributed in the processor cores behaves as a convex set. The sort objects of sort-middle rendering are the primitives transformed from the physical space into the screen space. Therefore, correct sorting of primitives can be achieved in the form of sampling points converted in the 3D screen space. However, global sampling and communication within sort-middle rendering, whether for opaque or semitransparent objects, can seriously affect parallel rendering performance.

Considering the fidelity and efficiency of multifield visualization, the proposed framework utilizes a hybrid sort-last and sort-middle parallel rendering scheme based on multiple visual channels. The framework introduced in this paper is shown in Figure 3, in which $\mathrm{G}$ represents the geometric processing and $\mathrm{F}$ represents the rasterization process. This scheme can separate opaque and transparent objects within the visualization pipeline, and then the most suitable parallel rendering method is selected according to the operation feature of visual fusion for each channel. Thus, accurate and scalable visualization of multifield data can be achieved.

\subsection{Hybrid Scheduling for Parallel Rendering}

The hybrid scheduling scheme can be represented as a two-way hybrid parallel rendering algorithm. The first way is the parallel rendering pipeline for the geometric channels that uses sort-last rendering, and the output pixel is used as part of the input data for the second way. According to the predefined conditions above, only opaque geometric objects that exhibit strong data locality for parallel rendering are processed in the geometric channels. Therefore, parallel rendering in the first way does not involve a sorting problem and it is independent of the complex mesh data characteristics of multiphysics simulations. Thus, the parallel rendering process in this way does not require data communication until the image is synthesized.

The second way is the parallel rendering pipeline in the optical channel. It adopts sort-middle rendering, in which the final subimage is generated locally in a processor core by communicating the sampling data of local mesh cells with each of the other processor cores. When the processor core completes data communication and performs local rendering, the original mesh cells will have been transformed into a series of sampling points in 3D screen space. At this point, the sorting problem between the subdomains in the original physical space is converted to a sorting problem between the sampling points in the 3D screen space. The sorting of the sampling points considers only the depth comparison test and it is also independent of the complex mesh data characteristics; therefore, there will be no ambiguity error regarding the sorting of scene 
objects. Thus, it can solve the parallel translucent rendering problem of complex domains in multiphysics simulations.

The two-way hybrid parallel rendering is performed during the ray-accumulation level of volume rendering within the optical channels. Since both the geometric and the optical channels can obtain linearized depth information in the rendered scene, the visual fusion between multiplex visual channels can be performed correctly. This hybrid scheduling scheme not only improves parallel visualization efficiency by taking advantage of data locality in the geometric channels, but it also provides accurate visualization results for the complex mesh data of multiphysics simulations. The hybrid parallel rendering introduced in this paper takes place in the uniform 3D sampling space; therefore, it can be used not only for CPU based rendering but also for GPU based rendering to meet the interactive analysis needs of different domain users.

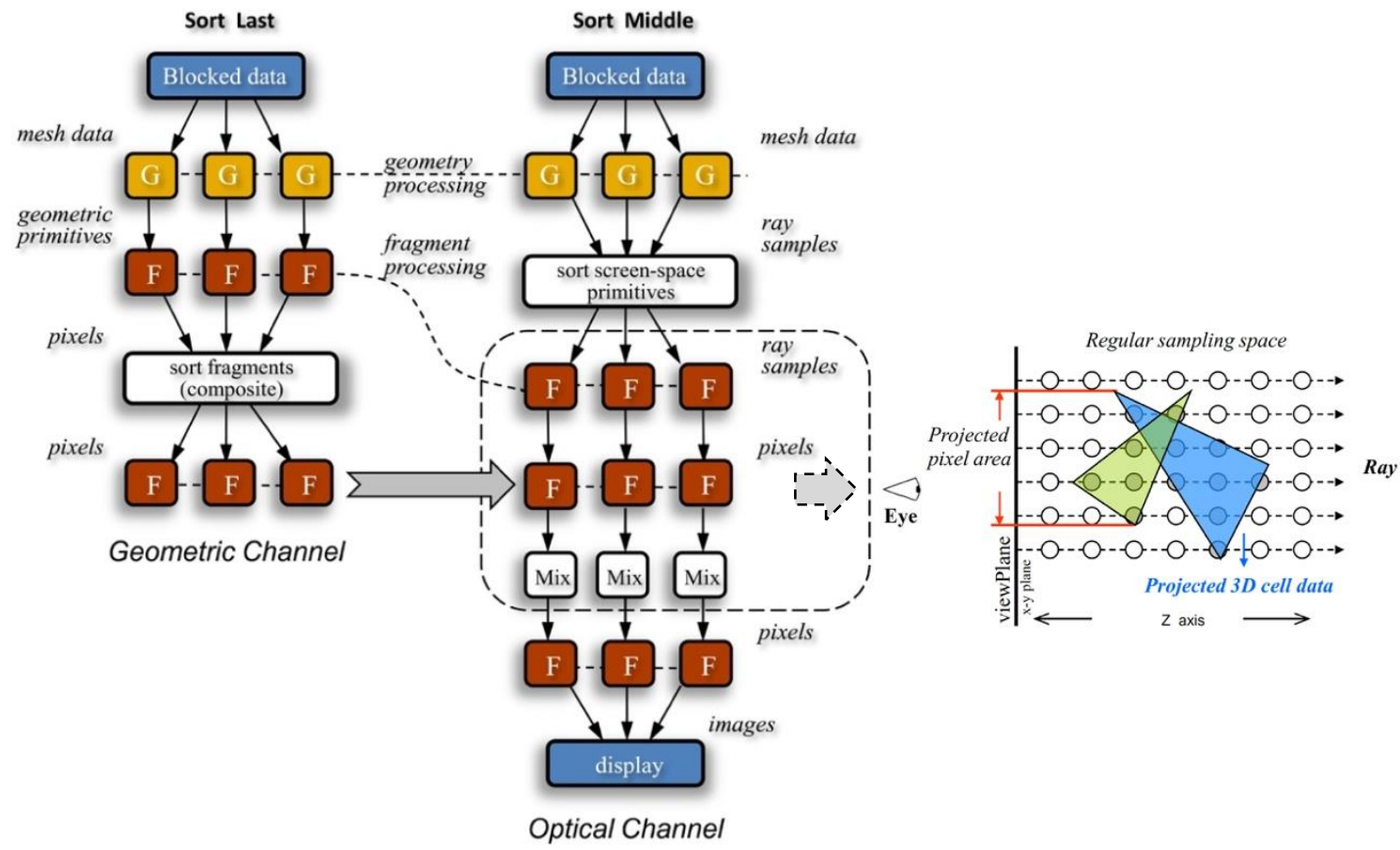

Figure 3. Hybrid scheduling for Parallel Rendering

\section{RESULTS AND DISCUSSION}

In this section, we discuss the application results of the proposed parallel visualization framework in several typical multiphysics simulation fields.

\subsection{Inertial Confinement Fusion}

Inertial confinement fusion (ICF) is controlled nuclear fusion that can provide a clean and sustainable energy supply for humans. This process involves cross-coupling relations among multiple physical processes. For example, one of the core scientific problems of ICF is laser-plasma instability or, more specifically, the mechanisms causing the instability and how best to control them. The cause of the instability is interaction between the laser beam and the plasma, which is affected by the coupling of multiple physical processes such as stimulated Raman scattering, stimulated Brillouin scattering, and the filament.

Currently, ICF radiation hydrodynamics 3D applications, based on the JASMIN parallel programming framework (Mo et al., 2010), can generate time-varying data sets of the scale of terabytes through fine physical modeling and simulation of billions of mesh cells. Without a method for multifield visualization that is intuitive and efficient for interactive data analysis, it will be difficult for ICF scientists to analyze and 
recognize the potential physical phenomena of interface instability. To visually analyze such multiphysics phenomena, traditional visualization (Figure 4c) uses a single-field geometric rendering method to represent the instability interface of the target region. However, because opaque geometry rendering can cause spatial structure occlusion, it is also necessary to use isovolumetric extraction to derive important information regarding the material interface. Nevertheless, it remains difficult to reflect fully the feature-rich trend of fluid interface in the original multiphysical field.

Compared with the results of traditional visualization, the multiple-CPU based multifield visualization supported by our framework could provide a more intuitive and clear representation of the interface instability for ICF domain scientists on $128 \mathrm{CPU}$ cores. As can be seen in Figure 4a, pseudocolor-based cross-section rendering is adopted in the geometric channels to represent the instability distribution in the axial center of the physical field. The volume rendering method is used in the optical channels to represent the 3D distribution of interface instability. Our multifield visualization framework could help ICF experts achieve rapid comparison of ensemble simulation results to deepen their understanding of hydrodynamic instability issues.

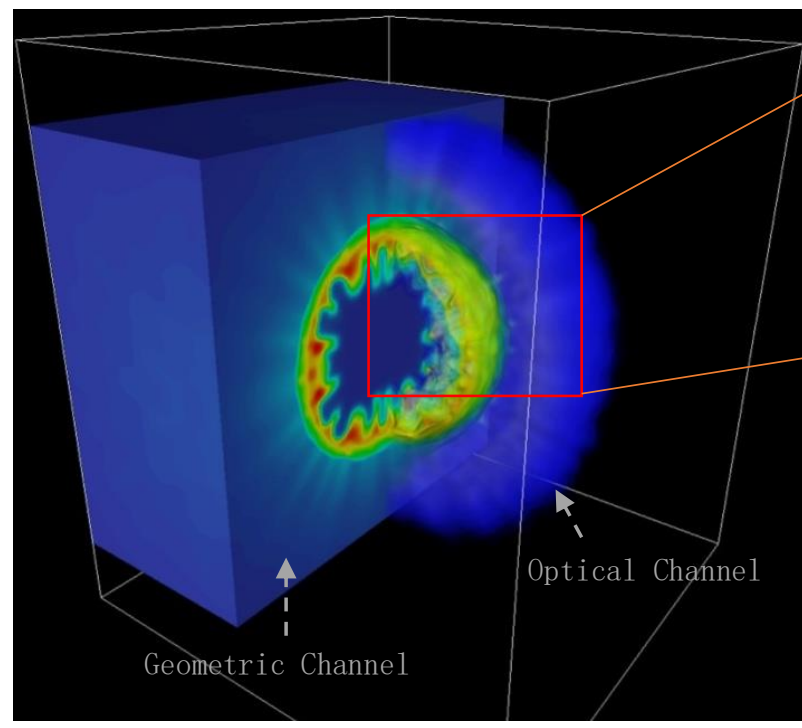

(a)Multiplex multifield visualization result

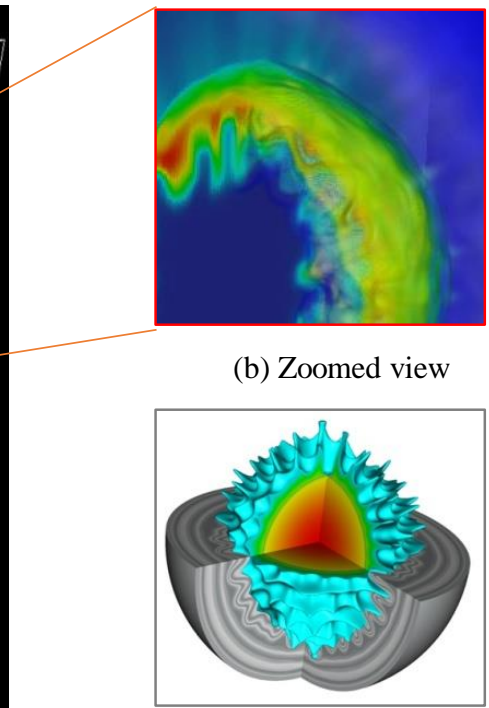

(c) Traditional visualization

Figure 4. Multiple-CPU based multifield visualization of fluid instability in ICF

\subsection{Electromagnetic Environment}

The EM environment refers to the overlapping of multiple EM signals distributed in the airspace, time domain, frequency domain, and energy domain, which has certain influence on both equipment and personnel. Computational EM modeling is a typical multiphysical techniques often used in the simulation of real-world EM applications, e.g., EM pulse radiation and scattering with irregular geometric structures.

Applications in the EM environment can be divided into three different scales of simulation: the device level, platform level, and regional level. Platform-level simulation is used mainly for multiscale electrical targets such as aircraft and ships. Regional-level simulation is used for regional scenes on the scale of square kilometers, and device-level simulation is used for components and devices on the meter-millimeter scale. Currently, simulations of platform- and regional-scale applications based on JASMIN can achieve the fine physical modeling and simulation of billions to tens of billions of mesh cells. EM experts need intuitive visualization methods to support the analysis of the very large-scale multifield data sets.

For the interactive analysis requirements of device-level EM applications, we adopt GPU-based multifield visualization using the multifield visualization framework proposed in this paper, and we realize real-time interactive visual analysis of high-power microwave simulation data using an NVIDIA k6000 graphics card. Figure 5 shows the multifield visualization results of tens of millions of particles, the electric field intensity and complex device models that satisfy the EM analysis demand of a device-level application. 


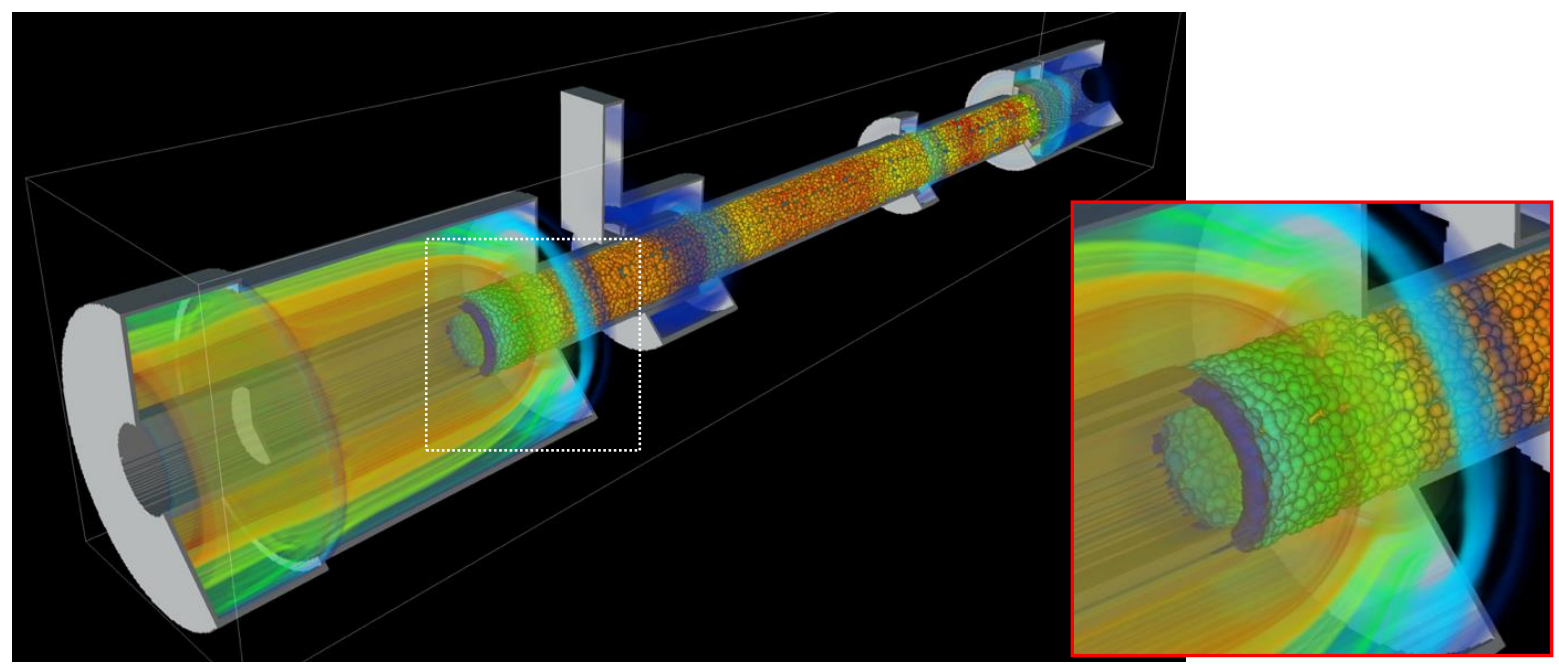

Figure 5. Hardware accelerated multifield visualization of device-level EM application

\subsection{Large-Scale EM Simulation Application}

An in situ visualization method (Cao et al., 2017) has also been employed for the ultra-scale simulation data of EM radiation shielding for an unmanned aerial vehicle with 8.5 billion mesh cells. This simulation used 8192 CPU cores of China's TianHe-2 supercomputer with a total simulation comprising 15,000 time steps and 187 time steps for the in situ visualization. The total time of the simulation and visualization was close to $120 \mathrm{~min}$. In terms of visualization, the in situ visualization of this example adopted the multifield visualization method presented in this paper. It completed the multifield visualization of three physical fields, including the aircraft model, cross-section surface of the 3D electric field, and energy distribution of the 3D electric field. The image resolution reached $1024 \times 1024$ pixels. The multifield visualization results of this application are shown in Figure 6. In this figure, geometric rendering of the model of the unmanned aerial vehicle and cross-sectional surface rendering of the energy distribution of the electric field was adopted in the geometric channels, and volume rendering of the energy distribution of the electric field was adopted in the optical channels. The multifield visualization framework proposed in this paper clearly reveals the wavefront distribution in the physical field, which would help accelerate the understanding of EM experts regarding EM mechanisms such as radiation and scattering.

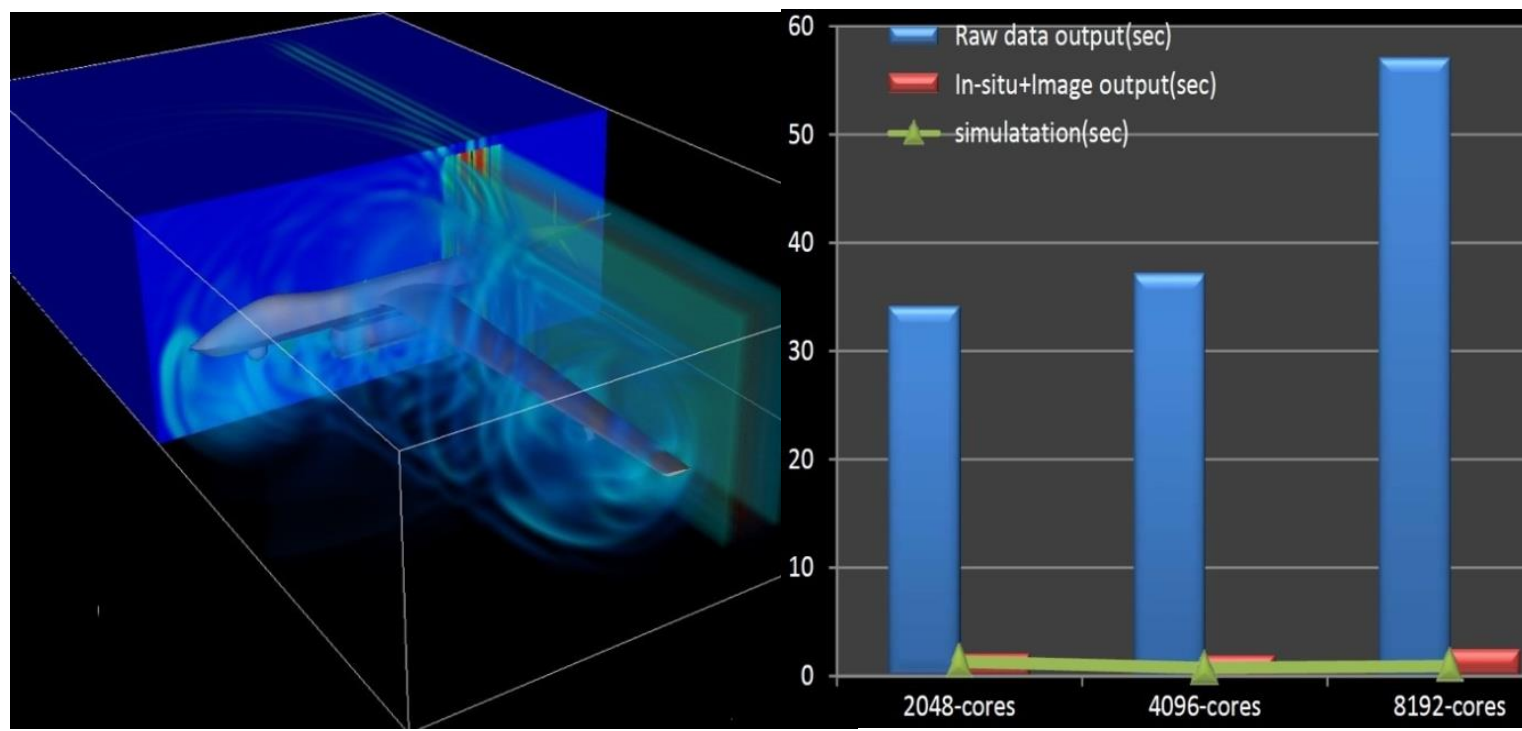

Figure 6. In situ visualization result (left) and scalable performance measurement (right) of the simulation of EM radiation shielding for an unmanned aerial vehicle on the Tianhe-2 supercomputer 
In terms of in situ visualization performance, it required $56 \mathrm{~s}$ per time step of the EM simulation to output the raw data to disk in the traditional off-line data analysis mode. Conversely, in situ visualization and image output only required $1.8 \mathrm{~s}$ per time step. Therefore, it can be seen that the efficiency of data analysis can be increased by at least 25 times by in situ visualization, demonstrating the scalability of our proposed hybrid scheduling scheme for parallel visualization of large-scale multiphysics simulations. The detailed test results of this large-scale simulation are presented in the right-hand panel of Figure 6.

\section{CONCLUSIONS}

In this paper, we proposed a hybrid scheduling framework for parallel visualization of computational multifield data, which can overcome the two fundamental problems of multifield visualization. A multiplex visual-channels-based multifield visualization scheme was introduced, which was used to solve the visual representation problem of complex multiphysics phenomena. A hybrid scheduling framework for parallel rendering was also presented, which was used for scalable visualization of large-scale multifield data. The framework has been applied in typical applications, e.g., fusion energy and EM environments. Compared with the traditional method, the visualization images obtained using our proposed framework are considered more intuitive and of higher fidelity. Our proposed parallel visualization scheme is scalable because it preserves the original mesh partitioning, making it suitable for applications involving large-scale multiphysics simulation and data analysis.

\section{ACKNOWLEDGEMENT}

This work was supported by the National Key R\&D Program of China under grant NO.2017YFB0202203, and the Defense Industrial Technology Development Program of China (Grant No. C1520110002).

\section{REFERENCES}

Bertin J.,1983. Semiology of graphics: diagrams, networks, maps. University of Wisconsin Press, London.

Cai W. et al, 1999. Data intermixing and multi-volume rendering. Computer Graphics Forum, 18(3):359-368.

Cao Y. et al, 2017. In situ visualization infrastructure for large scale simulations with structured meshes. International Conferences Computer Graphics, Visualization, Computer Vision and Image Processing. IADIS Press, Portugal, Lisbon, pp.139-146.

Eberly D.H., 2006. 3D Game Engine Design: A Practical Approach to Real-Time Computer Graphics, pp. 69. Morgan Kaufmann Publishers, United States.

HVS model, 2019. https://en.wikipedia.org/wiki/Human_visual_system_model

Insley J.A. et al, 2011. Viusalizing Multiscale, Multiphysics Simulation Data:Brain Blood Flow. IEEE symposium on Large-Scale Data Analysis and Visualization, pp. 3-7.

Jacq J. et al, 1997. A Direct Multi-Volume Rendering Method Aiming at Comparisons of 3-D Images and Models, IEEE Transactions on Information technology in Biomedicine, Vol.1, pp. 30-43.

Kreeger K.A. et al, 1999. Mixing translucent polygons with volumes. Proceedings of IEEE Visualization, pp. 191-198.

Lethbridge P., 2005. Multiphysics Analysis. The Industrial Physicist, udson, Ohio.

Molnar S. et al, 1994. A Sorting Classification of Parallel Rendering. IEEE Computer Graphics and Applications, 14(4), pp. 23-32.

Mo Z.Y. et al, 2010. JASMIN: A parallel software infrastructure for scientific computing. Frontiers of Computer Science in China, 4(4):480-488.

Phadke M.N. et al, 2012. Exploring Ensemble Visualization. Proceedings of SPIE. Vol. 8294, pp.1-12.

Porter T. et al, 1984. Compositing digital images. SIGGRAPH'84: Proceedings of the 11th annual conference on Computer graphics and interactive techniques. ACM Press, New York, NY, USA, pp. 253-259.

Ren J. et al, 2012. Composition Computation of Radiation Hydrodynamics and Particle Transport Based on JASMIN. Chinese Journal of Computational Physics, 29(2): 205-212. 